\title{
ANÁlisis DEL ELECTROLITO DEL ACUMULADOR AUTOMOTRIZ A DIFERENTES TEMPERATURAS EN CONDICIÓN DE ENCENDIDO
}

\author{
ANALYSIS OF THE ELECTROLYTE OF AN \\ AUTOMOTIVE ACCUMULATOR FOR DIFFERENT \\ TEMPERATURES IN STARTING CONDITION
}

\author{
Johnny Pancha ${ }^{1, *}$, Vicente Rojas ${ }^{1}$, Vicente Romero ${ }^{1}$, Jorge Nejer ${ }^{1}$
}

\section{Resumen}

Una batería automotriz del tipo plomo ácido es un elemento que genera una fuerza electromotriz capaz de abastecer de energía a todo el sistema eléctrico del vehículo. En el presente estudio se analiza el comportamiento de la densidad específica del electrolito durante la condición de arranque de un motor térmico; además, se considera como una variable, el gradiente de temperatura de funcionamiento que puede estar expuesto una batería automotriz. Obteniendo resultados de una variación inversamente proporcional del comportamiento de la temperatura del electrolito y su densidad en las celdas del acumulador durante la condición de arranque. Se concluye que las condiciones externas como la temperatura pueden afectar directamente a la densidad y las condiciones eléctricas de un acumulador, así como identificar el comportamiento de estas durante el funcionamiento en un vehículo.

Palabras clave: arranque, batería, electrolito, temperatura.

\begin{abstract}
An automotive battery of the acid lead type is an element that generates an electromotive force capable of supplying energy to the entire electrical system of the vehicle. In this investigation, the behavior of the specific density of the electrolyte during the start-up condition of a heat engine is analyzed; the operating temperature gradient at which the battery can be exposed is also considered as a variable. According to the results, the electrolyte temperature is inversely proportional to its density in the cells of the accumulator during the start-up condition. It is concluded that external conditions, such as the temperature, can directly affect the density and electrical conditions of an accumulator, and can identify the behavior of these during the operation in a vehicle.
\end{abstract}

Keywords: Starting, Battery, Leaf acid, Temperature.

\footnotetext{
${ }_{1, *}$ Facultad de Mecánica, Escuela Superior Politécnica del Chimborazo, Ecuador. Autor para correspondencia johnny.pancha@espoch.edu.ec. (D) http://orcid.org/0000-0001-7320-2154, (D) http://orcid.org/0000-0001-5658-3055, (D) http://orcid.org/0000-0003-2317-7071, (D) http://orcid.org/0000-0002-5744-2585.
}

Recibido: 15-11-2018, aprobado tras revisión: 17-12-2018

Forma sugerida de citación: Pancha, J.; Rojas, V.; Romero, V. y Nejer, J. (2019). «Análisis del electrolito del acumulador automotriz a diferentes temperaturas en condición de encendido». INGENIUS. N. ${ }^{\circ} 21$, (enero-junio). pp. 88-94. DOI: https://doi.org/10.17163/ings.n21.2019.09. 


\section{Introducción}

La batería automotriz ha sido diseñada para trabajar cuando un motor de combustión interna necesita encenderse mediante el uso de un motor de arranque y el acumulador automotriz; debe tener la capacidad para abastecer de energía durante esta condición para generar los primeros giros del motor. La condición de arranque es el comportamiento eléctrico donde la batería automotriz tiene el más alto desempeño y la correlación entre la densidad específica del electrolito del acumulador en función de la temperatura [1]. La densidad específica se relaciona con el estado de carga de una batería y la interacción con el material activo, la influencia de la temperatura se relaciona con el voltaje y la intensidad de corriente que esta puede entregar [2]. La variación en el gradiente de temperatura utilizado y la condición de arranque enfoca resultados que pueden relacionarse con los obtenidos en el estudio de la gasificación del electrolito de un acumulado durante su funcionamiento de carga y la temperatura expuesta [3]; la cual genera datos adicionales del comportamiento en una condición tan común durante el funcionamiento de un automotor. Es muy importante estudiar el efecto de la temperatura en el funcionamiento de las placas negativas y positivas, durante el proceso de carga, descarga, funcionamiento de arranque y condiciones externas como la temperatura ya que afectan su vida útil y, además, al estar expuesta a un electrolito como es el ácido sulfúrico [1].

\subsection{Batería automotriz}

Elemento primordial del sistema eléctrico del vehículo, diseñado para abastecer de energía entre algunas condiciones eléctricas siendo la más primordial la capacidad de arranque para cumplir el objetivo esencial de encender el motor [4]. La capacidad de arranque es efectiva en un acumulador durante el tiempo de 10 segundos y abasteciendo del máximo amperaje posible que puede entregar su diseño interno durante la condición eléctrica que se produce cuando se generan los giros del motor. Esta prueba, denominada condición de arranque, se ha estandarizado a una temperatura ambiente $25{ }^{\circ} \mathrm{C}$, mientras que la prueba realizada a $0{ }^{\circ} \mathrm{C}$ y $-18{ }^{\circ} \mathrm{C}$ se denomina condición de arranque en frío [5].

\subsection{Densidad específica}

En 1860, Gaston Plante combinó un $\mathrm{Pb} / \mathrm{PbSO}_{4}$ con un electrodo de $\mathrm{PbO}_{2} / \mathrm{PbSO}_{4}$ en una solución de ácido sulfúrico y obtuvo una fuente de energía electroquímica con gran fuerza electromotriz [4].

La temperatura es un factor importante que influye en la fuerza electromotriz de un acumulador, por lo que baterías automotrices expuestas a bajas temperaturas presentan problemas en su diseño interno impidiendo que puedan desarrollar su trabajo con normalidad, característica de los vehículos en regiones frías [6]. Mientras que la exposición del acumulador en condiciones de altas temperaturas acelera el deterioro de los elementos internos y acelera la oxidación de las placas positivas.

La resistencia eléctrica específicamente (resistividad) del electrolito $\mathrm{H}_{2} \mathrm{SO}_{4}$, es uno de los parámetros básicos que determinan la resistencia interna y el poder en cada una de las celdas de una batería plomo ácido [7]. Cuando la resistencia interna de la celda es alta, una parte sustancial de la energía útil se consume dentro de la propia celda, es decir, la energía se pierde en forma de calor, cuando la corriente eléctrica fluye a través de la celda, depende de la concentración y temperatura del electrolito [7].

\subsection{Temperatura}

Se considera que el electrolito $\left(\mathrm{H}_{2} \mathrm{SO}_{4}+\mathrm{H}_{2} \mathrm{O}\right)$ tiene $1,280 \mathrm{~g} / \mathrm{cm}^{3}$ de densidad, es decir, 1,280 veces más pesado que un volumen igual de agua pura mientras los dos líquidos están a la misma temperatura [8].

En el diagnóstico automotriz, la herramienta más útil y efectiva para conocer el estado de la densidad específica se denomina densímetro o hidrómetro [5]. Una densidad comprendida entre $1,290 \mathrm{~g} / \mathrm{cm}^{3} \mathrm{y}$ $1,270 \mathrm{~g} / \mathrm{cm}^{3}$ indica que el acumulador está completamente cargado, mientras que una densidad comprendida entre $1,240 \mathrm{~g} / \mathrm{cm}^{3}$ y $1,200 \mathrm{~g} / \mathrm{cm}^{3}$ muestra que la carga del acumulador se ha reducido. Cuando la densidad específica obtenida en el densímetro es inferior a $1,150 \mathrm{~g} / \mathrm{cm}^{3}$ se considera que la batería se encuentra descargada. Estos valores se refieren a una temperatura del electrolito comprendida entre $20{ }^{\circ} \mathrm{C}$ a $25{ }^{\circ} \mathrm{C}$ [5]. Por lo tanto, se debe aplicar un ajuste por cada 5 grados de variación de la temperatura del electrólito sobre $25{ }^{\circ} \mathrm{C}$ supone una corrección de la densidad relativa en 0,0035 unidades [5].

\subsection{Condición de arranque}

Durante la condición de funcionamiento de un motor térmico, el dispositivo fundamental es el motor de arranque porque es el encargado de generar los primeros giros del motor para que pueda encenderse [5]. En el sistema eléctrico se genera una condición particular del comportamiento de la tensión cuando el motor inicia el giro, lo que se puede observar en la Figura 1. La norma ISO16750-2 determina que la condición de arranque se estructura por la variación de tensión en valores que oscilan en relación del tiempo, que la norma estandariza en tiempos entre 3 a 10 segundos; tiempo en el cual existe alta demanda de intensidad de arranque lo que relaciona que la batería vaya descargándose por el trabajo realizado y esto se refleja en la densidad específica del electrolito [9]. 
La tensión del sistema eléctrico del vehículo decrece momentáneamente durante el arranque del motor. Este valor de tensión depende del estado de la batería. Los valores pueden bajar a distintos niveles entre $10,5 \mathrm{y}$ 10,9 voltios cuando el acumulador se encuentra en buen estado [5].

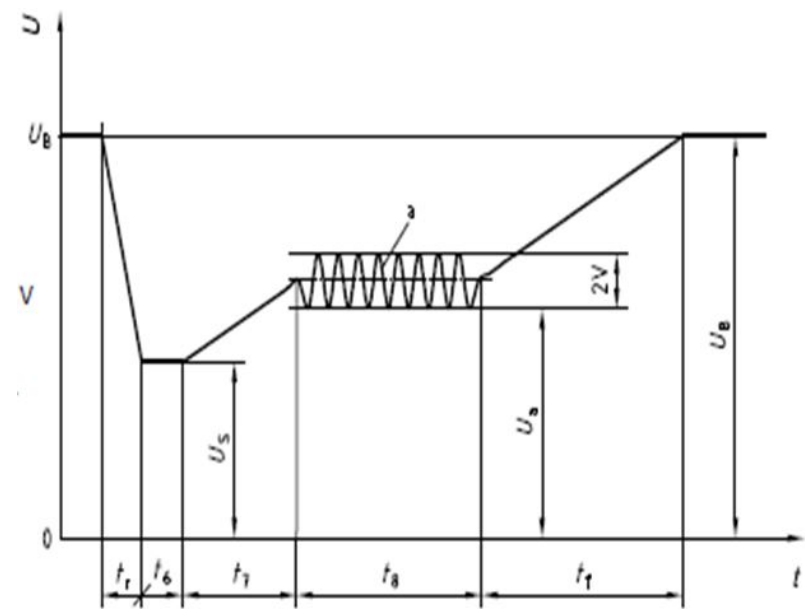

Figura 1. Comportamiento de la tensión de circuito eléctrico durante el arranque del motor térmico [4].

La temperatura es determinante en el desempeño del acumulador por lo que esta es mayor en altas temperaturas con relación a la capacidad de arranque en frío [9]. La resistencia eléctrica específica (resistividad) del electrolito $\mathrm{H}_{2} \mathrm{SO}_{4}$ es uno de los parámetros básicos que determina la resistencia interna y el poder de una célula de plomo ácido.

En un acumulador plomo-ácido, el sulfato es un sistema cerrado que se puede encontrar en las placas o en el electrolito según el estado de carga o la condición de funcionamiento. Si la batería está completamente cargada, entonces el sulfato está en el electrolito; mientras la batería está descargada, el sulfato está en las placas. El resultado final que presenta la densidad específica es una imagen que se relaciona con el voltaje y, por lo tanto, del estado de carga [9].

El objetivo de este análisis es conocer la relación del comportamiento de la densidad específica del electrolito de un acumulador automotriz durante la condición de arranque, generando una variación de la temperatura ambiente a la que está expuesta la batería. Por lo general el análisis de la densidad específica del electrolito se lo realiza para conocer el estado de carga, pero se busca analizar su incidencia cuando se somete al cambio ambiental de temperatura durante el proceso de almacenamiento y confirmar el cambio estructural en la densidad y su relación con la tensión e intensidad que ofrece la batería [10].

El estudio busca identificar el comportamiento de la densidad específica de la batería durante la condición de arranque, para conseguir indicios que puedan dirigir este análisis hacia elementos iniciales de investigación y futuras mejoras con relación al diseño de baterías automotrices. No existen estudios relacionados sobre el comportamiento de la densidad específica durante el arranque en baterías plomo ácido por lo que se desea obtener valores con base en la variación de temperatura.

\section{Materiales y métodos}

La investigación realizada basa su estudio en un método experimental el cual comprende el análisis del comportamiento de la densidad específica de una batería automotriz durante la condición de arranque bajo diferentes condiciones ambientales de temperatura. Para esto se han realizado mediciones de la densidad específica en las 6 celdas que conforman una batería automotriz en condiciones óptimas de funcionamiento, cuya de tensión nominal es de 12 voltios.

El proceso aplicado que se realiza es para determinar de manera práctica el estado de una batería ante el arranque de un motor. Para comenzar el estudio se obtiene el valor de la densidad específica de cada una de las celdas para obtener su valor condición de reposo inicial; se genera el arranque del motor para así comprobar la condición de estabilidad del electrolito para lo que se repite dicha prueba 6 veces continuas sin llegar a encenderlo y se procede a registrar el valor de la densidad específica de cada celda al final de dichas pruebas. Es importante mencionar que se registra la temperatura del electrolito antes y después de la prueba para posteriormente aplicar el ajuste necesario de la densidad específica en relación con la temperatura de transición.

\section{Resultados y discusión}

\subsection{Condición de temperatura alta}

La temperatura externa de la batería se incrementa progresivamente para lo que se utiliza un calefactor térmico simulando temperatura ambiente. Se procede a registrar el valor de densidad específica de cada una de las celdas de la batería. En la Tabla 1 se observa el registro de los datos obtenidos a $20{ }^{\circ} \mathrm{C}$ y de los valores de ajuste con base al cálculo en función de la temperatura [5].

Los valores de ajuste de la densidad se realizan con base al porcentaje de pérdida y dilatación, pero durante el proceso se hace el análisis con los valores ajustados y los obtenidos en cada prueba y medida realizada.

En la Figura 2 se aprecia el comportamiento de la densidad sobre la base de la estabilidad de temperatura a $55^{\circ} \mathrm{C}$; que indica un cambio grande en el primer bloque de celdas, pero una mayor estabilidad sobre 
todo en las celdas centrales, y con una estabilidad más alta en el grupo de celdas cercanas al borne negativo.

Tabla 1. Densidad y temperatura antes del arranque
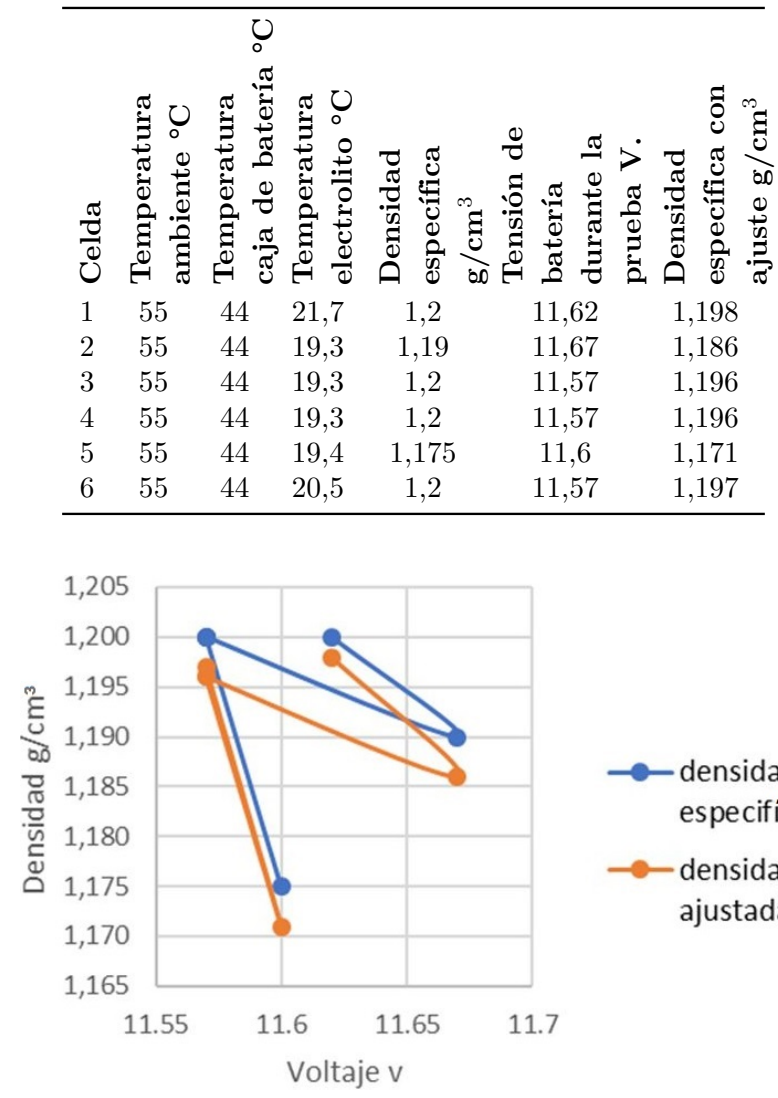

$\multimap$ densidad

especifíca

$\longrightarrow$ densidad ajustada

Figura 2. Gráfica de comportamiento de la densidad antes de someter al arranque a $55{ }^{\circ} \mathrm{C}$.

En la Tabla 2 se muestra los resultados obtenidos después de realizar el proceso de arranque continuo para ver la condición de estabilidad del acumulador y de los valores de ajuste con base al cálculo en función de la temperatura [5].

Tabla 2. Densidad y temperatura antes del arranque

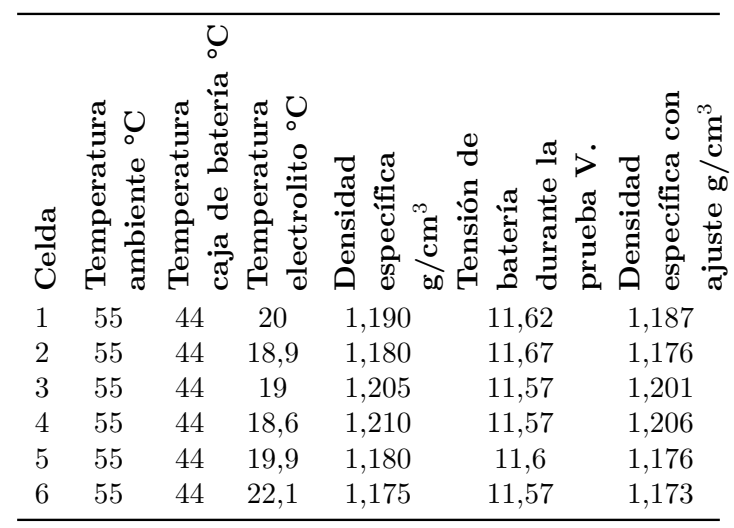

En la Figura 3 se aprecia el comportamiento de la densidad sobre la base de la estabilidad de temperatura de $55{ }^{\circ} \mathrm{C}$, manteniendo estable la tensión de la batería al momento de generar el proceso de consumo en arranque. Dando como resultado un cambio notable en el primer bloque de celdas, aunque su estabilidad se ve estable en las celdas centrales, pero generando un cambio notorio en las celdas cercanas al borne negativo.

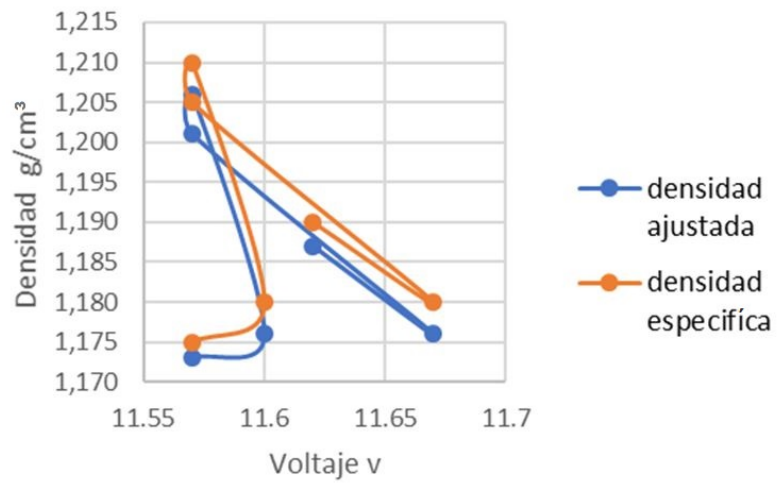

Figura 3. Gráfica de comportamiento de la densidad luego de someter al arranque a $55^{\circ} \mathrm{C}$.

\subsection{Condición de temperatura baja}

Para el registro de los datos en condiciones de temperatura baja, la cual se consigue encerrando a la batería en una cápsula de hielo seco hasta bajar su temperatura hasta el valor indicado; las celdas se someten a un cambio de temperatura inferior a la prueba anterior, logrando estabilizarla en una temperatura de $1^{\circ} \mathrm{C}$. En la Tabla 3 se muestran los valores obtenidos y de los valores de ajuste con base al cálculo en función de la temperatura [5].

Tabla 3. Densidad y temperatura antes del arranque

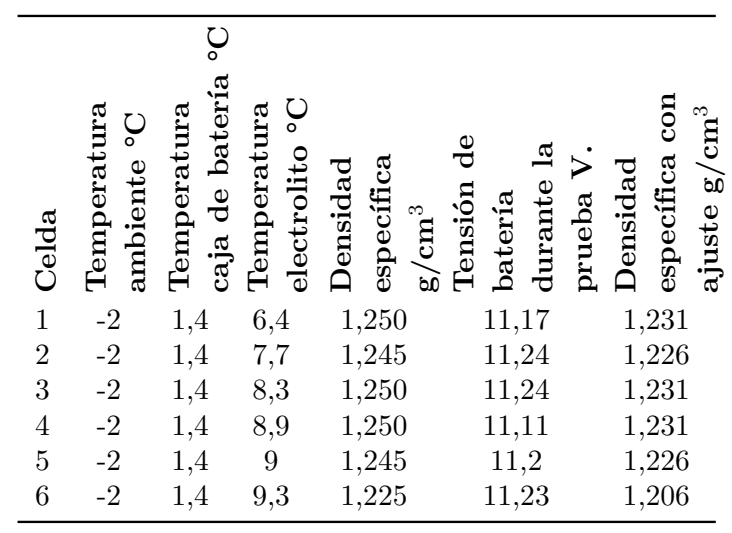

En la Figura 4 se observa el comportamiento de la densidad sobre la base de la estabilidad de temperatura de $-2{ }^{\circ} \mathrm{C}$ : el comportamiento del electrolito a temperaturas bajas es más estable con respecto al de temperaturas altas. 


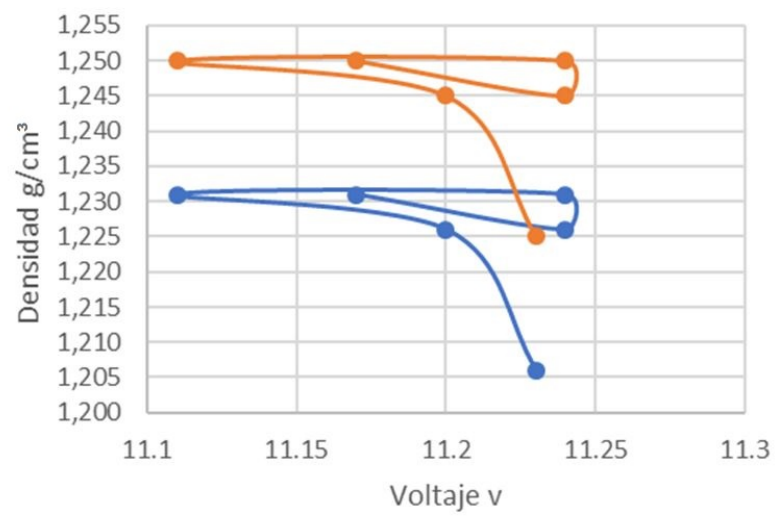

$\longrightarrow$ densidad ajustada densidad especifica

Figura 4. Gráfica de comportamiento de la densidad antes de someter al arranque a $-2{ }^{\circ} \mathrm{C}$.

En la Tabla 4 se muestra los resultados obtenidos después de realizar el proceso de arranque continuo para ver la condición de estabilidad del acumulador y de los valores de ajuste con base al cálculo en función de la temperatura [5].

Tabla 4. Densidad y temperatura antes del arranque

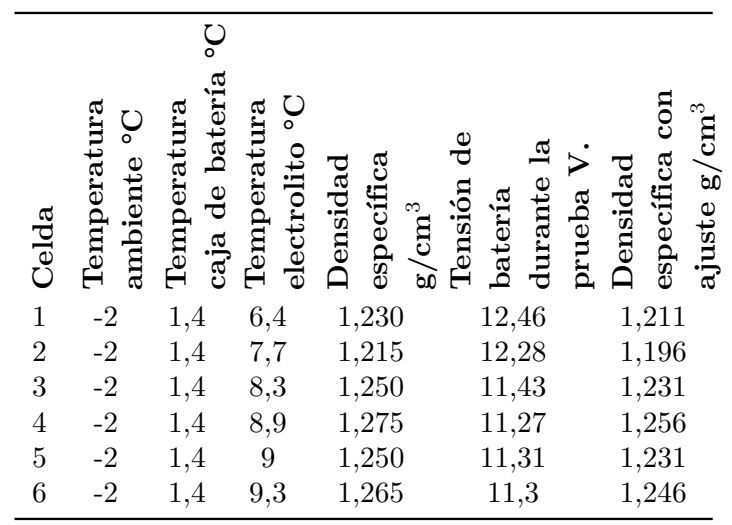

En la Figura 5 se muestra el comportamiento de la densidad sobre la base de la estabilidad de temperatura de $-2{ }^{\circ} \mathrm{C}$, luego del proceso de arranque, cuyos valores de caída de densidad electrolítica y caída de tensión son considerables más aún en el grupo de celdas del borne positivo, indicando una minoría en la reacción y un esfuerzo en el proceso de descarga de esta durante dicha fase de prueba.

\subsection{Condición de temperatura ambiente}

Con el proceso establecido en los casos anteriores, se obtienen los datos a temperatura ambiente de $27{ }^{\circ} \mathrm{C}$. En la Tabla 5 se observan los valores de densidad específica y temperatura obtenidos con valor de ajuste [5].

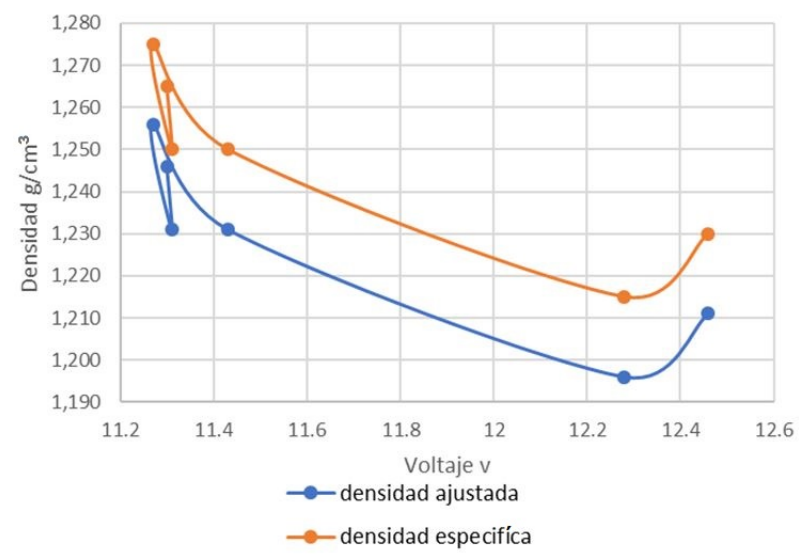

Figura 5. Gráfica de comportamiento de la densidad después de someter al arranque a $-2{ }^{\circ} \mathrm{C}$.

Tabla 5. Densidad y temperatura antes del arranque

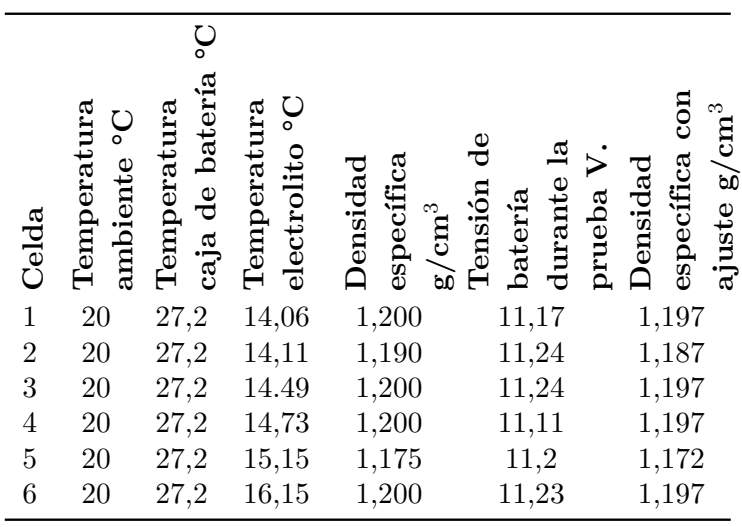

En la Figura 6 se observa el comportamiento de la densidad sobre la base de la estabilidad de temperatura de $20{ }^{\circ} \mathrm{C}$, indicando una estabilidad constante de la tensión y densidad de la batería.

Además, se puede observar un comportamiento de flujo cíclico por parte de la densidad del electrolito, a más de una estabilidad en la tensión de la batería, la cual se mantiene estable sin importar la condición de arranque.

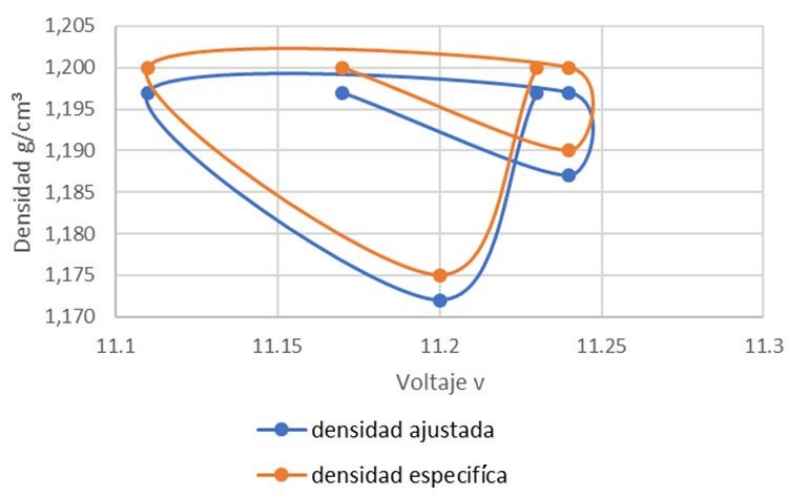

Figura 6. Gráfica de comportamiento de la densidad después de someter al arranque a $20{ }^{\circ} \mathrm{C}$. 
Los resultados se ven comparados en la prueba de arranque para obtener un análisis del comportamiento de la tensión y densidad de la batería bajo condiciones extremas lo que da un porcentaje de cambio de temperatura ambiente con respecto a condiciones altas de $0,3900 \%$ y de $3,1375 \%$ en temperaturas bajas indicando que la densidad del electrolito se ve afectada más por condiciones bajas, pero en tiempo estimado de arranque y estabilidad de tensión por las condiciones de temperatura alta como se puede apreciar en la Figura 7.

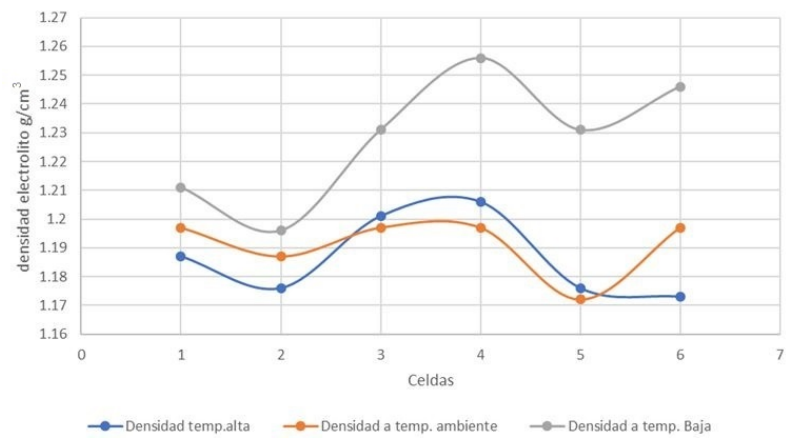

Figura 7. Gráfica de comparación de densidad a diferentes temperaturas.

\section{Conclusiones}

Las figuras representan el cambio de la densidad y tensión cuando se genera un cambio de temperatura y estabilización. Además, cada punto de la gráfica representa una celda y muestra el comportamiento del electrolito en las mismas.

La Figura 2 indica el valor de la tensión de la batería antes de generar el proceso de consumo en arranque. La estabilidad del acumulador en condiciones funcionamiento se comprueba tanto en voltaje y densidad.

La Figura 3 indica que el cambio de la tensión de la batería no es muy considerable al momento de generar el proceso de consumo en arranque, pero la alteración de la densidad del electrolito se ve alterada sobre todo en las celdas centrales.

Realizando un promedio del funcionamiento de las celdas, la tensión nominal sigue estando estable.

La Figura 4 indica el valor de la tensión de la batería antes de generar el proceso de consumo en arranque. Se observa una estabilidad en las condiciones de funcionamiento del acumulador en su densidad, mas no en su tensión.

La Figura 5 indica que el cambio de la tensión de la batería es muy considerable al momento de generar el proceso de consumo en arranque, pero la alteración de la densidad del electrolito tiene un cambio insignificante con respecto a las pruebas anteriores, sobre todo en las celdas finales. Esto indica que la caída de estabilidad en las condiciones de funcionamiento del acumulador se ve más afectada cuando se procede a realizar el proceso de arranque a temperaturas bajas.

La Figura 6 indica que el cambio de la tensión de la batería no es muy considerable al momento de generar el proceso de consumo en arranque, pero la alteración de la densidad del electrolito se ve alterada de manera mínima en todas las celdas. La caída de estabilidad de las condiciones en el funcionamiento del acumulador se ve más afectadas cuando se procede a realizar el proceso de arranque a temperatura ambiente.

La Figura 7 nos presenta los resultados del comportamiento de la densidad a diferentes temperaturas y que al tener temperaturas más bajas se estabiliza más la densidad, pero pierde fuerza en la condición de arranque, mientras tanto en caliente se tiene más fuerza de arranque, pero la pérdida de tensión es mucho mayor.

El comportamiento de la densidad del electrolito es directamente proporcional al cambio de temperatura. Cuando aumenta la temperatura a valores más altos registrados en el funcionamiento del habitáculo del motor, la estabilidad se mantiene.

La densidad de la batería se ve alterada en su condición estable, sobre todo en las celdas centrales y finales al bajar la temperatura considerablemente, debido al recorrido de los electrones entre ellas provocando una caída brusca de tensión, pero su recuperación es mucho más rápida debido a que la densidad del electrolito se estabiliza.

$\mathrm{Al}$ aumentar la temperatura, la tensión se mantiene constante, pero se efectúa una variación extrema en la densidad del electrolito durante el arranque. Esto afecta al funcionamiento y durabilidad de la batería, pues la vida útil de esta se reduce considerablemente debido a la variabilidad de la densidad.

Al bajar la temperatura del electrolito a valores mínimos de funcionamiento real, genera un cambio extremo en la tensión de la batería, generando un mayor esfuerzo de los consumidores principales en el proceso de arranque, lo que provoca daños en los componentes del vehículo.

$\mathrm{Al}$ disminuir la temperatura del electrolito los valores la intensidad decae debido a la dificultad del desplazamiento de electrones, sobre esforzando a los consumidores eléctricos y electrónicos a su funcionalidad, lo que nos generaría una mejora al diseño de la estructura de las baterías.

Sobre la base de los resultados estudiados se considera que la temperatura del electrolito en diferentes condiciones ambientales genera un cambio en la funcionalidad, recomendando el uso de nuevos materiales y diseños para mejorar el comportamiento de la batería durante la condición de arranque. 


\section{Referencias}

[1] J. E. Torres, A. Sierra, D. Y. Peña, I. Uribe, and H. Estupiñán, "Velocidad de corrosión de una aleación base plomo en una solución de ácido sulfúrico a diferentes temperaturas," Materia (Rio de Janeiro), vol. 19, pp. 182-196, 062014. [Online]. Available: https://goo.gl/tZqkW2

[2] A. Zolfaghari and G. Jerkiewicz, "Temperaturedependent research on $\operatorname{pt}(111)$ and $\operatorname{pt}(100)$ electrodes in aqueous $\mathrm{H}_{2} \mathrm{SO}_{4}$," Journal of Electroanalytical Chemistry, vol. 467, no. 1, pp. 177-185, 1999. [Online]. Available: https://doi.org/10.1016/S0022-0728(99)00084-4

[3] C. A. Cadena and R. F. Farfán, "Sensor de gaseo para baterías de plomo-ácido: estudio preliminar," Asociación Argentina de Energías Renovables y Ambiente; Avances en Energías Renovables y Medio Ambiente, vol. 14, pp. 175-182, 2010. [Online]. Available: https://goo.gl/m9Ug3G

[4] D. Pavlov, Lead-Acid Batteries: Science and Technology: A Handbook of Lead-Acid Battery Technology and Its Influence on the Product. Elsevier, 2017. [Online]. Available: https://goo.gl/WMEFcn
[5] J. M. Alonso, Técnicas del automóvil equipo eléctrico. Thomson Paraninfo, 2005. [Online]. Available: https://goo.gl/4p4ymo

[6] D. Diemand, Automotive Batteries at low temperatures. US Army Corps of Engineers, 1991. [Online]. Available: https://goo.gl/uWhBMV

[7] D. Pavlov, A. Kirchev, M. Stoycheva, and B. Monahov, "Influence of $\mathrm{H}_{2} \mathrm{SO}_{4}$ concentration on the mechanism of the processes and on the electrochemical activity of the $\mathrm{Pb} / \mathrm{PbO}_{2} / \mathrm{PbSO}_{4}$ electrode," Journal of Power Sources, vol. 137, no. 2, pp. 288-308, 2004. [Online]. Available: https://doi.org/10.1016/j.jpowsour.2004.06.006

[8] ISO, Road vehicles - Environmental conditions and testing for electrical and electronic equipment - Part 2: Electrical loads (ISO 160750-2), International Organization for Standardization Std. [Online]. Available: https://goo.gl/nSXN3w

[9] Megger, Battery testing guide. Megger, 2017. [Online]. Available: https://goo.gl/5RopkA

[10] E. Rojas, "Análisis de la densidad específica del electrolito de un acumulador de energía automotriz plomo ácido a diferentes temperaturas," Sectei, vol. 5, no. 1, pp. 80-84, 2018. 